\title{
In-situ study of structural evolution of tribological materials using synchrotron radiation
}

\author{
Vladimir Burov ${ }^{1, *}$, Ivan Bataev ${ }^{1}$, and Alexander Smirnov ${ }^{1}$ \\ ${ }^{1}$ Novosibirsk State Technical University, Materials Science Department, 630073, 20 Karl Marx Ave., Novosibirsk, Russia
}

\begin{abstract}
In this paper, we propose an approach for estimation of structural changes at the surface of metallic materials during running-in stage of friction. The application of synchrotron microbeam for in-situ observation of structural evolution in thin layers subjected to friction load is substantiated. A design of experimental apparatus intended for in-situ studies of friction processes using synchrotron radiation is described. This apparatus maintains constant position of the sample surface with respect to the incident beam. The ring shaped experimental sample with a thickness of $1 \mathrm{~mm}$ is pressed with constant pressure against a pin shaped indenter and rotated with constant speed. The pressure on the sample is determined by the mass of the load placed in the loading basket. The surface of the sample that comes out of indenter is probed by synchrotron beam. The proposed experimental scheme allows receiving X-ray diffraction patterns even under condition of severe plastic deformation of the surface. The preliminary results on the synchrotron assisted study of the dry friction of a $\mathrm{Cu}$ sample are described.
\end{abstract}

\section{Introduction}

One of the main reasons for the quality loss of engineering products used in movable joints is the initial stage of wear that leads both to a change in the dimensions and roughness and to a change in the structural state of the surface layers. X-ray diffraction study of the worn surfaces provides a valuable insight on the irreversible processes of structural evolution and formation of thin surface layers with a gradient structure $[1,2]$. Knowledge of sequence of rapid structural changes in the surface layers during the initial stages of sliding friction is important to predict the reliability and lifetime of the movable joints of mechanisms. The process of structural evolution during running-in stage (from initial state to the steady irreversible state) can be investigated using synchrotron x-ray diffraction [3].

\section{The objectives of the study}

Synchrotron diffractometry stations, which have a wide range of applications for study of rapid processes, differ in intensity of radiation, beam diameter and provide variety of universal devices that enable recording the diffraction patterns by $1 \mathrm{~d}$ and $2 \mathrm{~d}$ detectors.

It is known, that the process of dry friction is accompanied by the structural changes of the material surface layers [1-4]. This process leads to formation of the nanostructured layer, which substantially determines lifetime of parts of the movable joints. The application of the synchrotron radiation for tribological studies allows understanding the mechanisms of wear of materials with different chemical and phase compositions at different loads and friction speeds. In ref. [5] one can find a description of an experiment, when synchrotron radiation was used to probe the surface of D16 (Al-Cu-Mg) alloy during friction against high speed steel indenter. In ref. $[6,7]$ an application of synchrotron radiation for in-situ study of steel SUJ2 - sapphire friction pair is described. Both these experiments provided interesting insight on mechanisms of structural evolution during the friction.

It should be noted, that when the synchrotron beam is focused to $1-5 \mu \mathrm{m}$ spot the precise positioning of the friction surface with respect to the incident beam becomes extremely important since the friction leads to plastic deformation and wear. Thus, the position of the beam on the sample surface may change during the friction test. This is especially important in grazing-angle geometries or similar diffraction schemes.

The design of apparatus used by Kajita et. al. [6,7] provided high precision positioning and allowed using a microscope to get the images of the friction surfaces during experiments. This was provided by usage of transparent sapphire counterpart. However, one can rarely meet sapphire in real friction pairs. In our study, we developed a friction apparatus that allows application of different materials of both a sample and a counterbody (indenter). Fig. 1 represents the arrangement of the surface layer being investigated with respect to the X-ray beam. The unit is equipped with a control system that allows setting the rotation speed of the sample within the range of $0 . .5 \mathrm{~s}^{-1}$ and any number of rotation cycles, as well as the remote control (Fig. 2). In addition, a computer with control interface can be connected via a remote control.

* Corresponding author: v.burov@,corp.nstu.ru 


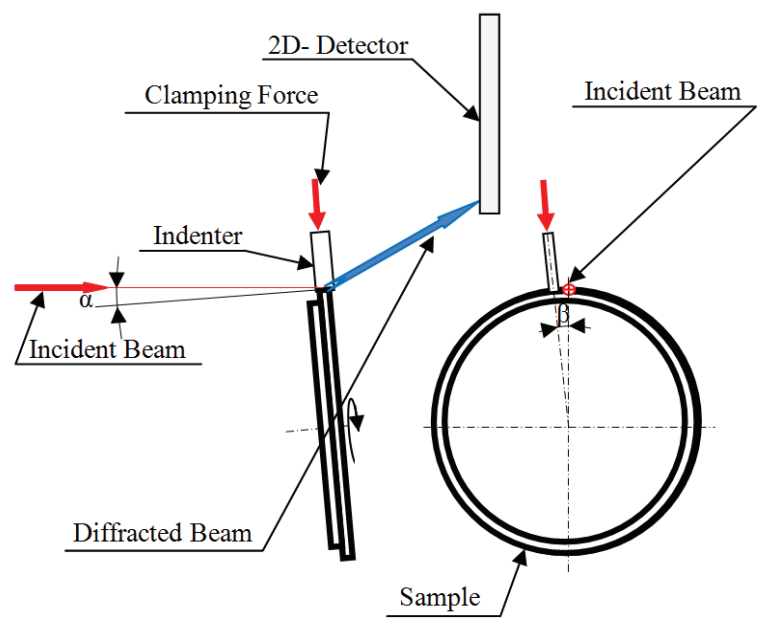

Fig. 1. The arrangement of a rotating sample and an indenter with respect to a sliding X-ray beam

This setup is designed to be used at microfocus beamlines of European Synchrotron Radiation Facility (ESRF) with diameter of the incident beam in the range $1 \ldots 3 \mu \mathrm{m}$. The apparatus is designed in such way, that the surface of indenter is kept at constant position during the tests. The sample is pressed against indenter with the required load. The sample has a shape of a disc with 1$\mathrm{mm}$ width rim on the outer curved or circular surfaces.

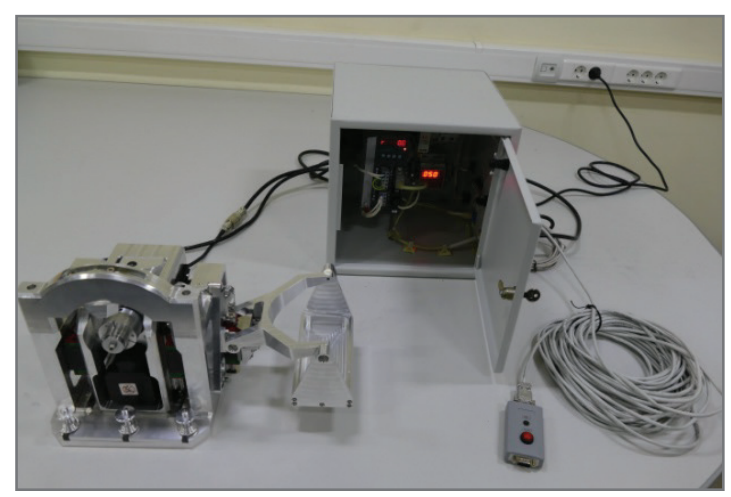

$\mathrm{a}$

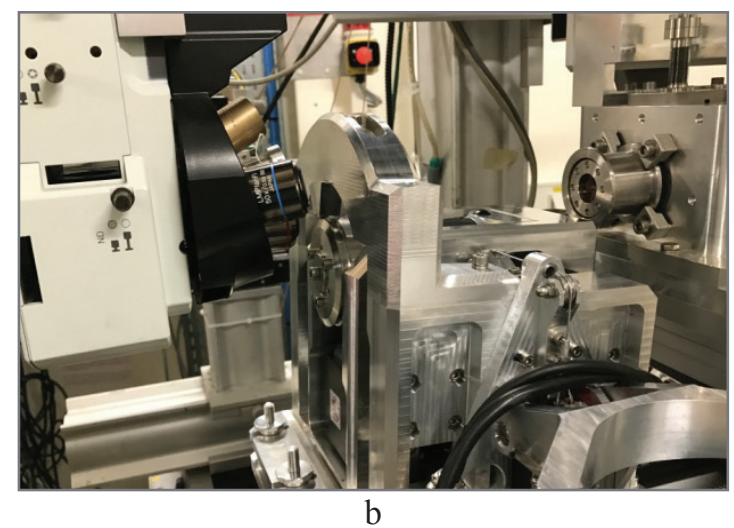

Fig. 2. Experimental apparatus designed for in-situ synchrotron assisted studies of rapid structural evolution that occurs at the surface layers during dry friction. (a) Overall view of the apparatus together with the control unit. (b) The apparatus placed at the beamline ID13 at ESRF.
The diameter of the sample should be in the range 60$70 \mathrm{~mm}$. The working area of the indenter has dimensions of 1 × $5 \mathrm{~mm}$ (Fig. 3). In the initial moment of time the sample is placed in such way that its contact area with the indenter is $1 \mathrm{~mm}^{2}$ (the possible increase of the contact surface due to the plastic deformation of the sample is neglected). Due to the fact, that the sample is pressed against the indenter and the pressure is kept constant during experiment, the relative arrangement of the incident beam and the sample stays the same during experimental time providing that indenter doesn't wear during the whole time of the experiment.

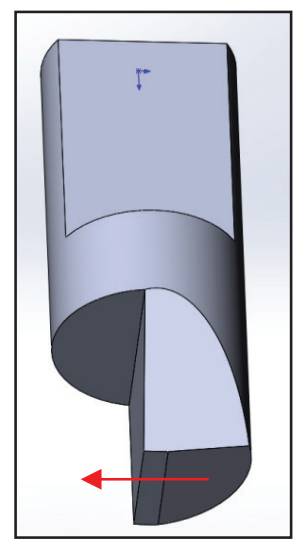

Fig. 3. The geometry of the indenter. The arrow shows the direction of the sample rotation.

The friction load is provided by test weights that are placed in the loading basket. The basket is attached to the body of apparatus via a lever. The body of tester moves inside the frame using low friction linear guides. In order to avoid shadowing of diffraction pattern by indenter the probing position is shifted from the friction position $2-8^{\circ}$ in direction opposite to the sample rotation direction. This leads to 2-20 ms temporal delay between the friction moment and the probing moment.

The experimental apparatus allows using 2D X-Ray detector Eiger $4 \mathrm{M}$ at ESRF beamline 13. This provides frequency of $750 \mathrm{~Hz}$ of data acquisition with exposition time less than $2.6 \mathrm{~ms}$.

\section{Results of preliminary experiments}

The friction of a copper sample against a high-speed steel indenter was studied using the apparatus to test new experimental technique. The copper discs were annealed in a furnace at $850{ }^{\circ} \mathrm{C}$ to minimize initial strains and provide a nondeformed surface. After the heat treatment, the sample was chemically polished to remove the oxide layer. The load on the sample was $40 \mathrm{MPa}$ and the rotation speed was $60 \mathrm{rpm}$. A total number of the sample revolutions during the time of the experiment was 5500 . The exposition time of detector was equal to the period of one revolution. Thus, first X-ray diffraction pattern contained information from all points of the sample subjected to friction during one revolution. The second frame contained information from all points of the sample that experienced friction load twice. And so on. The angle between the incident beam and the sample surface was $5^{\circ}$. 
The examples of diffraction patterns received during the experiment are shown in Fig. 3. One may notice, that the diffraction patterns, received in static conditions (Fig. 4a and $4 \mathrm{c}$ ) have sharp diffraction spots, which correspond to a polycrystalline material with limited amount of rather large individual grains. However, the diffraction rings after the experiment became more uniform and less spotty indicating that plastic deformation of the surface layer occurred leading to grain refinement. The diffraction rings, received in dynamic conditions were rather uniform (Fig. 3b). Since the exposition time was quite long a lot of individual grains passed under the beam and no sharp spots could be observed. One may also notice that only half of the rings are seen on the figure. The lower parts of the rings are absent due to the reflection geometry of diffraction, when lower part of the diffraction cones are absorbed by the sample.

Fig. 5 shows integrated diffraction patterns in coordinates "azimuthal angle - 20 ". The diffraction rings can be indexed as 111, 200,220 and 311 as it is typically observed on diffraction patterns of $\mathrm{Cu}$.

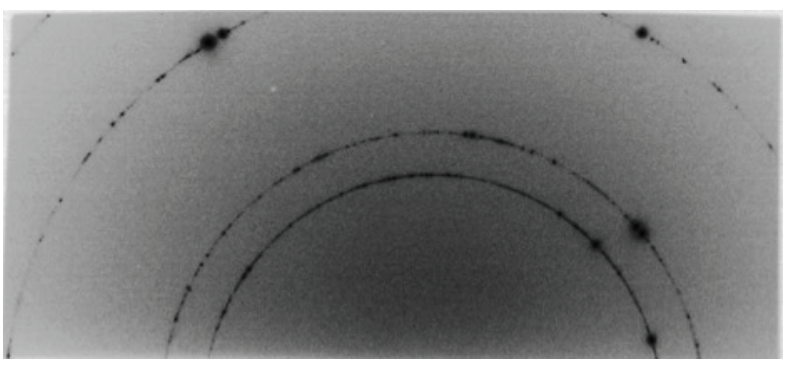

$\mathrm{a}$

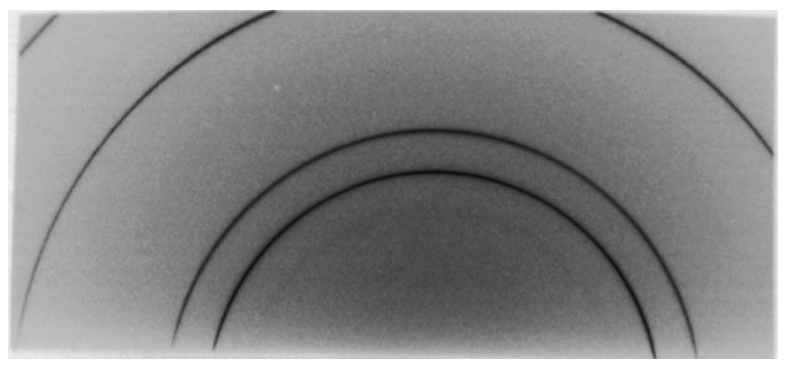

b

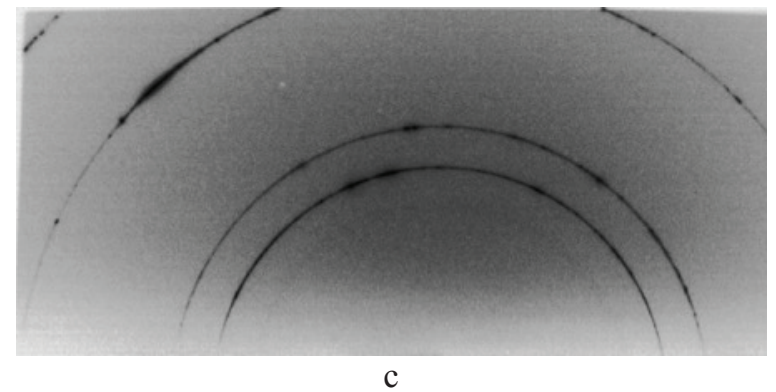

Fig. 4. X-ray diffraction patterns obtained from the sample surface in (a) static condition before the experiment, (b) in dynamic condition during the experiment and (c) in static condition after the experiment.

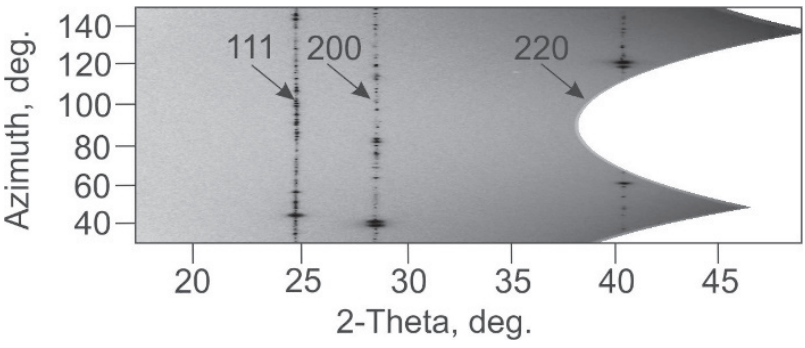

a

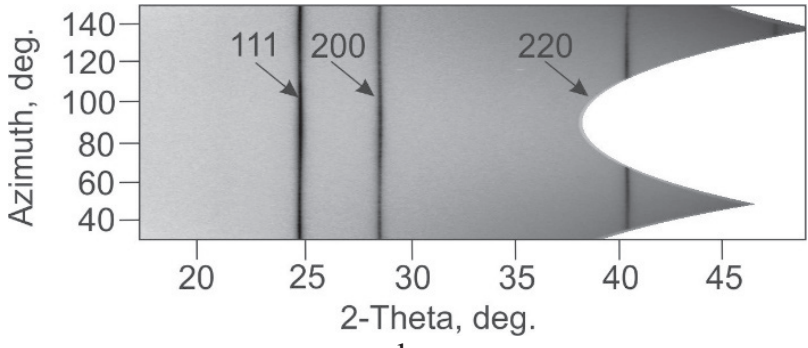

b

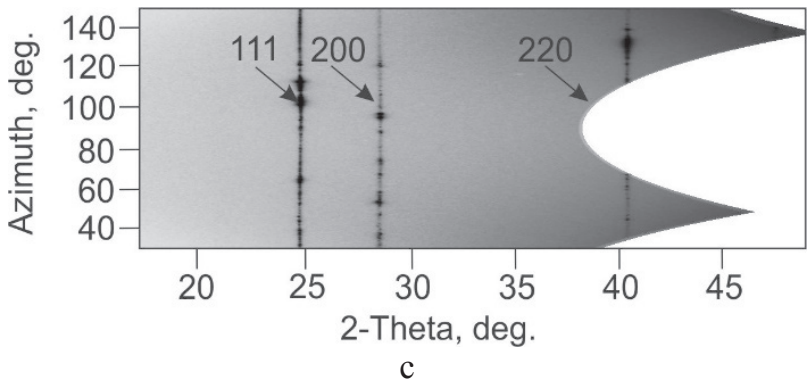

Fig. 5. Diffraction patterns of $\mathrm{Cu}$ sample (a) before the beginning of the experiment (b) during the experiment and (c) after the end of experiment.

Fig. 6 shows variation of the 111 peak intensity with the time of the experiment. One may observe that the intensity has noticeably decreased indicating possible plastic deformation and structural transformation at the surface layer. One may also observe broadening of 3 peaks of $\mathrm{Cu}$ (Fig. 7) indicating possible grain refinement and accumulation of defects and corresponding microstrains.

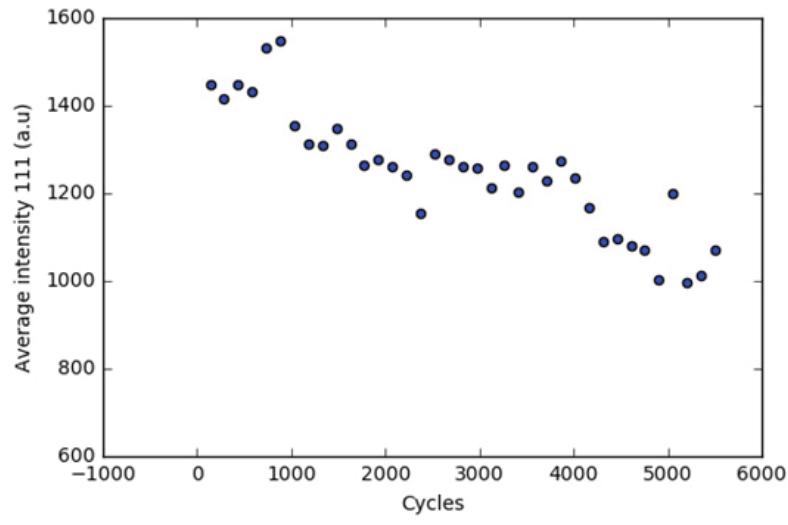

Fig. 6. Average intensity of 111 peak of $\mathrm{Cu}$ during friction against steel indenter. 


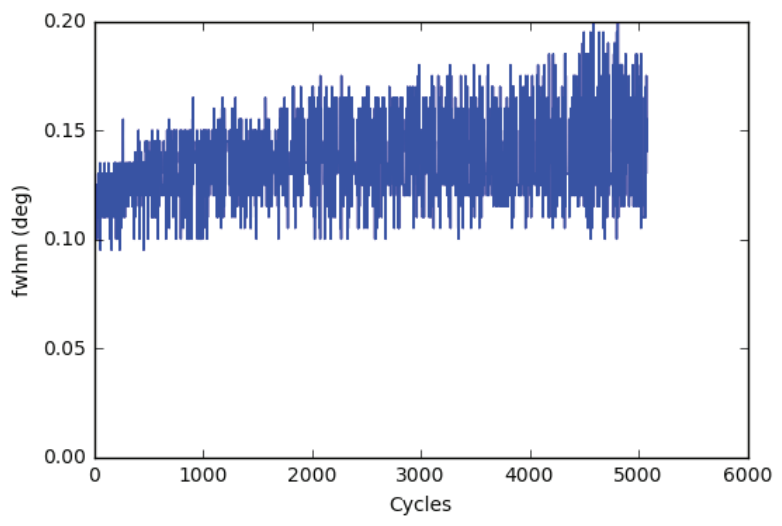

a

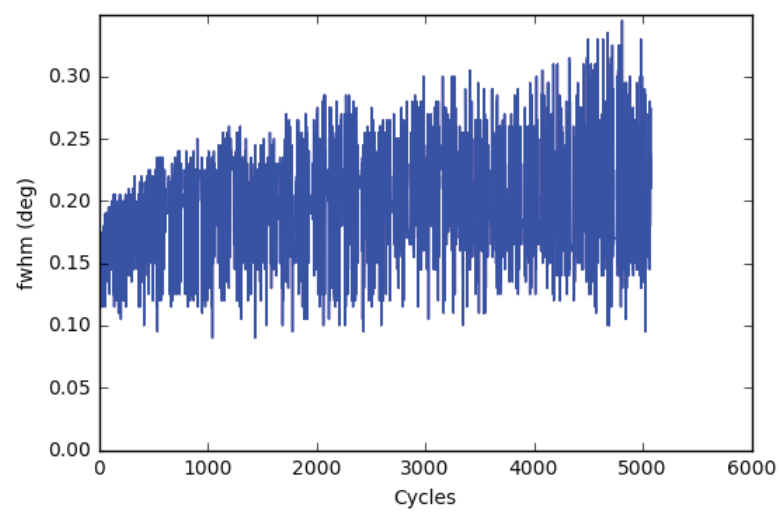

$\mathrm{b}$

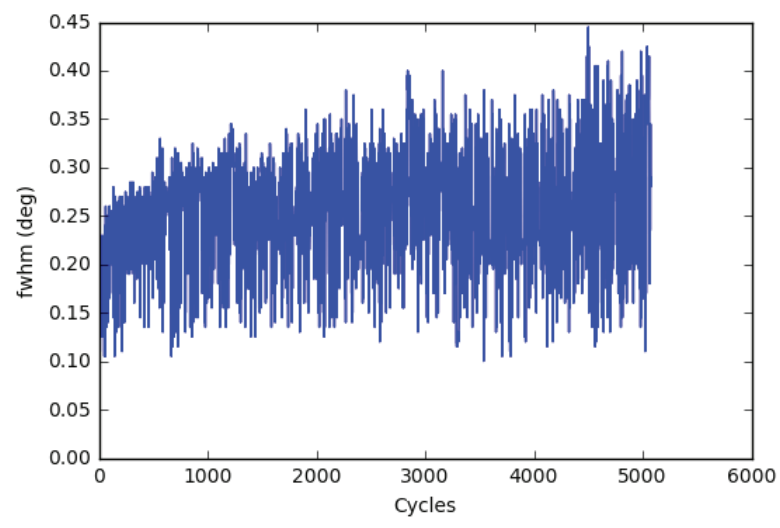

c

Fig. 7. Broadening of (a) 111 , (b) 200 and (c) 220 peaks of $\mathrm{Cu}$ versus number of friction cycles.

\section{Conclusion}

A novel approach to investigate the friction processes using synchrotron assisted diffraction was proposed. This approach allows probing the sample and receiving information about surface structure of materials under friction "on the fly". Using the copper-steel friction pair we showed that during the friction there are noticeable changes in the peaks intensities and widths which is due to accumulation of defects and structure refinement. In the nearest future, the algorithm will be developed that will allow retrieving the information about most important structural characteristics of materials (coherent scattering region size, microstrains, microstresses, dislocation density, etc).

This research was carried out using financial support of the Ministry of Education and Science of Russian Federation (program "Investigations and developments in the priority areas of the scientific and technological complex of Russia in 20142020”), agreement № 14.586.21.0026, unique identifier of agreement RFMEFI58616X0026.

\section{References}

1. A.V. Makarov, P.A. Skorynina, A.L. Osintseva, A.S. Yurovskikh, R.A. Savrai. Obrabotka metallov (tehnologiya, oborudovanie, instrumenty), 69, 80-92 (2015)

2. N.N. Soboleva, A.V. Makarov, I.Yu. Malygina. Obrabotka metallov (tehnologiya, oborudovanie, instrumenty), 4, 79-85 (2013)

3. V.P. Kuznetsov, A.V. Makarov, S.G. Psakhie, R.A. Savrai, I.Yu. Malygina, N.A. Davydova. Physical mesomechanics, 17, 250-264 (2014).

4. A.V. Kolubaev, O.V. Sizova, E.A. Kolubaev. Tribologie und Schmierungstechnik, 55, 45-48 (2008)

5. A.A. Bataev, I.A. Bataev, V.G. Burov, S.V. Burov. Russian Physics Journal, 59, 314-316 (2016)

6. S. Kajita, K. Yagi, T. Izumi, J. Koyamachi, M. Tohyama, K. Saito, J. Sugimura. Tribol. Lett, 57, 11 (2015)

7. S. Kajita, T. Izumi, J. Koyamachi, M. Tohyama, K. Saito, J. Sugimura. Tribol. Lett, 61, 16 (2016) 